Monatsschrift f. Geburtshülfe u. Gynäkologie 1922;57:223-234

\title{
IV. Literatur-Verzeichnis
}

\section{Geburtshilie.}

Aubert, Der transperitoneale Kaiserschnitt am unteren Segment. Rev.

franc, gyn. obst. 1921. März. Baer, Verrenkimg des Sakro-lliakalgelenkes. B. Johns Hopk. H. XXVIII.

Nr. 315. Baumm, H., Osteogenesis imperfecta. Arch. f. Gyn. 115. H. 2. S. 38ס. Bigler, Zur

Pathogenese der Schwangerschafttoxikosen. Schweiz. med.

Woch. 1920. Nr. 43. Bozsán, Experimentelle Beiträge zur Pathologie der Eklampsie. Orvosi Hetil. 1920. Nr. 39 u. 40. Broaha, Muß man den abdominalen Kaiserschnitt unbedingt für reine Fälle aufsparen ? Gyn. obst. 1920. Nr. 6. Cleisz, Koxalgische Beckeń (vergleichende Messungen beider Becken-

hälften). Bull. mém. soc. anat. Paris. 1920. Nr. 4. Cocke und Mason, Behandlung der akuten Appendizitis während der letzten

Schwangerschaftswochen. Kaiserschnitt und Appendektomieheil·ung.

J. Am. m. Ass. 1920. 10. XII. Deucher, Beitrag zur Kasuistik der Lungenembolie ingraviditate. Zbl. f. Gyn.

3. XII. S. 1743. Esau, Tetamï,s nach Abort und Douglaseiterung. Zbl. f. Gyn. 26. XI.

S. 1695. Fekeie, Nierenerkrankung und Schwangerschaft. Orvoskys. 1921. Festschrift f. Tauffer. -, Fuchs, Molnar fun., Die Schwangerschaftsnierenerkrankungen. Orvoskys.

1921. H. 1. Frey, Der abdominale Kaiserschnitt in Lokalanästhesie. Munch, med.

Woch. 2. XII. S. 1548. Genge, Eklampsie und Kaiserschnitt. Brit. m. J. Nr. 3130. Genschel, Zur

Kasuistik des Nabelschnurbruches. Zbl. f. Gyn. 3. XII.

S. 1750. Gessner, Die baclische Eklampsiestatistik für das Jahr 1919 im Lichte der Diätetik. Zbl. f. Gyn. 10. XII. S. 1814. Grosse, Wiederholter Kaiserschnitt bei einer achondroplastischen Frau.

Rev. de gyn. obst. 1921. März.

224

Literatur-Verzciebnis.

Groíe, Versache über Keimesänderung durch Sekreteinfluß. Dtsch. med.

Woeh. 1. XII. S. 1461. Haim, Bin zystisches Lymphangiom des Halses als schweres Geburtshindernis. Zbl. f. Gyn. 19. XL S. 1664. Hamburger, Über das Befinden von 1·ungentuberkulösen Frauen, bei denen eine Unterbrechung der Schwangerschaft abgelehnt wurde. Zbl. f.

Gyn. 26. XI. S. 1685. Hammerschlag, Die wesentlichen Bestimrmmgen im Entwurf des rieuen preußischen Hebammengesetzes. Dtsch. med. Woch. 24. XI. S. 1432. Hans, H., Decapitatio obliqua, Kaisersehnitt nnd temporäre Tubensterilisation. Med. Klin. 23. X. S. 1298. Heimann, Zur Kaiserschnittsfrage. Zbl. f. Gyn. 26. XI. S. 1700. Heil, Ein Fall von Thorakopagus. Prakt. Arzt. 6. Jahrg. 3/4. 21. Hellendall, Zur Behandlung des fieberhaften Abortes. Zbl. f. Gyn. 8. X.

S. 1448. -, Ein Fall von Schwangerschaft nach Sterilisierung mittels doppelter 
Unterbindung und Durchsehneidung beider Eileiter. Med. Klin.

11. IX. S. 1116. Henrotay, Indikation der abdominalen Hysterektomie während der Geburt ohne Beckenanomalie. Gyn. Obst. IV. H. 4. S. 336. Herring, Die Wirkung der

Schwangerschaft auf Größe und Gewicht ver-

schiedener Organe. B. in. J. Xr. 3128. Hieß und Beckmann, Zur Pathologie und Klinik der

Xierenerkrankungen

in der Schwangerschaft. Zbl. f. Gyn. 10. XII. S. 1773. Hinselmann, Über das Öclem cler

Schwangeren. Zbl. f. Gyn. 24. IX.

S. 1361. Hirsch, Über den Einfluß der obligatorischen Prophylaxe und Anzeige-

pflicht der Ophthalmoblennorrhoea neonatorum. Munch, med. Woch.

23. IX. S. 1223. Hirsch, M., Das ärztliche Heiratszeugnis, seine wissenschaftlichen und

praktischen Grundlagen unter Mitwirkung von Posner, Westenhvfer.

Czellíizer, Leppmann, Heller, P. Siraßmann, Sonntag. C. Kabilzsch.

Leipzig 1921. Hirst, Geburtshilfliche Todesfälle. J. Am. m. Ass. 29. X. S. 1406. IIofbauer, Zur Klärung der Eklampsiefrage. Zbl. f. Gyn. 17. XII. S. 1797. Hofer, .Encephalitis lethargica und C1/8aviditas. Zbl. f. Gyn. 5. XL S. 1604. Hiissy, Kurzes Repetitorium der Gebvutshilfe. 2. Aufl. 1922. E. Birscher,

Bern. Holmes, Geburtshilfliche Täuschungen. Eine Betrachtung über pseudo-

wissenschaftliche Bestrebungen in der modernen Geburtshilfe. Am.

J. obst. gyn. II. 3. S. 225. Bespre. ebenda S. 297. Hunter, Über die eklamptische Toxin. Brit. m.

J. 1920. S. 3092. Jaschke, v., Die Behandlung des fieberhaften Abortus. Zbl. f. Gyn. 5. XL

S. 1589. Josephsson, Über schwierige Perineotomie. Hygiea. 1918. S. 1195. Jehenhäuser, Uterushemie in einer rupturierten Kaisersclmittsnarbe.

Dtsch. med. Woch. 8. IX. S. 1050. Jung, Schnellentbindung durch vaginalen Kaiserschnitt bei

Grippe-

pnetimonie. Schweiz. med. Woch. 20. S. 728.

Literatur-Verzeichnis.

225

Kaboíix, Symphyseneiterung in der Schwangerschaft. Zbl. f. Gyn. 24. IX.

S. 1367. Kaíz, Über den plötzlichen ïod in Schwangerschaft, Geburt und Wochen-

bett. Arch. f. Gyn. 115. H. 2. S. 283. Kautzky, Die Benennung der Nierenerkrankungen in der Schwangerschaft.

Zlb. f. Gyn. 10. XII. S. 1765. Kehrer, Über Tuberkulose und Schwangerschaft. Munch, mecl. Woch.

16. IX. S. 1171. Kehrer, Der Wert der Beizdiagnose bei der Geburtsleitung. Munch, med.

Woch. 23. IX. S. 1208. Kirstein, Über eine auffallende biologische Eigentümlichkeit der Neugeborenen. Dtsch. med. Woch. 17. XI. S. 1393. -, Gegen den Dämmerschlaf in der Geburtshilfe. Westdeutsche Ärzte-

zeitung. 11. Jahrg. Xr. 2. -, Die prognostische Bedeutung der Keimhämolyse bei Kreißenden und

Wöchnerinnen. Arch. f. Gyn. 115. H. 2. S. 313. -, Über die passive Immunisierung des

Neugeborenen mit v. Behrings

Diphtherievakzin „T. A.” Arch. f. Gyn. 115. H. 2. S. 326. Klaar, Zur Xomenklatur der geburtshilflichen Wendung. Wien. klin. Woch.

15. IX. S. 452. Klee, Zur Technik der Ent·fernung expirierter Massen beim asphyktischen 
Neugeborenen. Med. Klin. 18. IX. S. 1142. Kraízeisen, Tierversuche mit Plazenta. Opten. Dtsch. med. Woch. 20. X.

S. 1260. Kritzler, H., Arbeiten über Prolapsoperationen (1916-1920). (Fortsetzung aus Xr. 20 d. Z.) 16. X. S. 1271. -, Beobachtungen über das Vorkommen von Diphtheriebazillen und

diphtheroiden Stäbchen beim Xeugeborenen unter besonderer

Berücksichtigung der klinischen Bedeutung dieses Befundes. Z. f.

Gyn. u. Geb. LXXXIV. H. 1. S. 179. - , Zur Hebung der Asepsis in der Xeugeborenen- und Wöchnerinnen-

pflege. Dtsch. med. Woch. 20. X. S. 1263. -, Fall von Prolapsus vaginae et uteri et intra partum mit tödlichem

Ausgang für Mutter und Kind. Zbl. f. Gyn. 3. XII. S. 1753. -, Allerlei Wissenswertes aus dem geburtshilflich-gynäkologischen Schrift-

tum. Med. Klin. 4. XII. S. 1487. -, Student und Gummihandschuh. Zbl. f. Gyn. 26. XII. S. 1703.

Kiistner, Zur Frage der Apnoe und Asphyxie der Kaiserschnittskinder.

Zbl. f. Gyn. 1. X. S. 1415. Landau, Zur Kenntnis der Streptokokkensepsis. Dtsch. med. Woch.

8. IV. S. 1061. Langer, Über Sirenenbildung. Z. f. G. u. Gyn. LXXXIV. H. 1. S. 151. de Lee, Ein Messer für den zervikalen Kaiserschnitt. Surg. Gyn. obst.

Oct. 21. S. 431. Liepmann, Die Größenbestimmung des äußeren Muttermuncles in der

Geburt. Zbl. f. Gyn. 10. IX. S. 1289. i $\Lambda$ epmann und Schulz, Xeue Ergebnisse zur Plazentar- und Eklampsie-

forschung. Dtsch. mecl. Woch. 24. XL S. 1417. Liepmann, Beitrag zuv Benutzung von

Schlingen und Gurten in der prak-

220̈

Literatur-Verzeichnis.

tischen Geburtshilfe (nebst Beschreibung der Lampendochtschlinge

des Kephalokoi·taspator). Munch, med. Woch. 8. XII. S. 1586. Liepmann, Ätiologie und

Behandlung der Eklampsie. Zbl. f. Gyn. 17. XII.

S. 1810. Lönne, Über Aplasia cutis congenita, Zbl. f. Gyn. 19. XI. S. 1653. Loschi, Die Therapie zur Behebung der Beckenfehler mit bleibender Wirkung

durch Exzision des oberen Teils der Schoßfuge (Partialsymphysi-

ektomie Coster). Zbl. f. Gyn. 22. X. S. 1523. Lorenzen, Über einen Fall von Salvarsanvergiftung in der Schwangerschaft

mit tödlichem Ausgang im Wochenbett. Zbl. f. Gyn. 1. X. S. 1407. Lynch, Die

Beekenartikulationen während Geburt und Puerperium. Surg.

gyn. obst, XXX. Nr. 6. Mahnert, Über die Anwendung eines neuen Hypophysenpräparates

„Pítrin” in der Geburtshilfe. Med. Klin. 13. XI. S. 1388. Mathes, Mutterschutz und.

Schwangerenfürsorge. Wien. klin. Rundsch.

20. X. S. 126. Mayer, A., Die Kiellandsche Zange. Zbl. f. Gyn. 29. X. S. 1557. Meyer-Rüegg,

Ein besonderer Befund bei einer Plazentarlösung. Schweiz.

med. Woch. 1920. S. 326. Meyer-Rüegg, Gravidität bei Uterusseptus. Zbl. f. Gyn. 24. IX. S.

1364. Moníuoro, Die Behandlung der Tympania uteri. Rivista d' ost. ginec.

pratica. III. Nr. 7. S. 265. Morriss, Die Bedeutung des Blutzuckers vom Standpunkt des Geburtshelfers, mit besonderer Bezugnahme auf den plazentaren Austausch.

B. Johns Hopkins H. Vol. XXVIII. Nr. 914, Miindheim, Hyperemesis gravidarum durch Salvarsan geheilt, Berl. klin. 
Woch. 5. XII. S. 1437. Mosse, Aminosäurestickstoff des Blutes in Fallen von normaler und komplizierter Schwangerschaft und beim Neugeborenen. B. Johns Hopkins Hosp. XXVIII. Nr. 316. Nacke, Spätgeburten. Med. Klin. 6. XL S. 1356.

Xassauer, Die schmerzlose Geburt. Munch, med. Woch. 21. X. S. 1364. Nebel, Über das Verhältnis von Aborten zu Geburten in Mainz in dem letzten Dezennium. Zbl. f. Gyn. 19. XL S. 1657. Nevermann, Über Narkolepsie in der Schwangerschaft, Dtsch. med. Woch.

29. IX. S. 1164. Novak, Zeitliche und kausale Beziehungen zwischen Geburt, Ovulation und Menstruation. Zbl. f. Gyn. 26. XL S. 1697. Nürnberger, Über die Verwendbarkeit der renalen Schwangerschaftsglykosurie zur Frühdiagnose der Gravidität. Dtsch. med. Woch.

22. IX. S. 1124. Cosentinó, Fünflingsgeburt in der königl. Frauenklinik in Palermo. Riv. d'ostet. ginec. prat, III. Nr. 9. S. 397. Oetiker, Anna, Vier Fälle von Sectio caesarea cervicalis in kleinsten klinischeri

Verhältnissen. Schweiz. med. Woch. 51. Jahrg. Nr. 22. Otrich, Hypophysenextrakt in Verbindung mit Lokalanästhesie. J. Am.

m. As. Nr. 9. 1921. Oeüingen, Kj. v., Beitrag zur Genese tier Schwangerschaftstoxikosen, insbesondere der Eklampsie. Zbl. f. G. 22. X. S. 1510.

Literatur-Verzeichnis. 227

di Palma, Interstitielle Tubarschwangerschaf t: 2 Fälle. Surg. gynec.

obst. Sept. S. 285. Peterfi, Der gegenwärtige Stand der Physiologie der Geschlechtsbestimmung. I. Progame Faktoren. Dtsch. m. W. 20. X. S. 1265. II. Syngame

Faktoren. 27. X. S. 1299. III. Metagame Faktoren. 3. XI. S. 1332.

IV. Schluß. Dtsch. med. Woch. 10. XI. S. 1363. Phillips, Kel·ichhnsten im Anschluß an Geburt. 2 Fälle. Am. J. med.

sciences. Febr. 21. Pilsky, Darmnekrose im 9. Schwangerschaftsmonat. Zbl. f. Gyn. 19. XI.

S. 1662. Plaß, Plazentare Übertragung. Kreatinin und Kreatin im Gesamtblut

und Plasma von Mutter und Fötus. B. Johns Hopkins H. XXVIII. Polak, Gewaltsame

Entbindung: ihre Stellung im geburtshilflichen Unter-

richt. Am. J. obst. gyn. II. 3. S. 237. Besprechung ebenda. S. 297. Polak, Beobachtungen über ektopische Schwangerschaften. (Studie an

307 Fallen.) Am. J. obst. gyn. II 3. S. 280. Bespr. ebenda. S. 326. Potrin, Hysterektomie bei akuter Puerperalinfektion. Gyn. obst. IV.

H. 3. S. 207. Precechtel, Die Bedeutung des Chinin beim Abortus. Casop. lék. ceskych.

Xr. 15. 21. Pribram, Über einen Verblutungstod bei Abort. Zbl. f. Gyn. 15. X.

S. 1482. Puppel, Über die innere Sekretion der Plazenta. Dtsch. med. Woch.

27. X. S. 1294. Raefler und Schultze - Pvhonhof. Die Hypnose bei vaginalen Kursunter-

suchungen Schwangerer. Zbl. f. Gyn. 10. IX. S. 1270. Remy, Die Gefahren der Phlatitis während der Schwangerschaft. Rev.

Mem. de gyn. et d'obst. Juli 1919. Remy, Plötzlicher Tod von Embolie bei einer schwangeren Frau. Rev.

franc, de gyn. et d'obst. Nr. 4. 1920. Rißmann, Geburtshilfliche Ratschläge für den Praktiker.

Dtsch. med. Woch

22. IX. S. 1135. Rißmann, Bedarf der praktische Arzt für die Geburtshilfe eines selbsthaltenden Aortenkompressorium? Munch, med. Woch. 21. X. S. 1304. Rosenthal, N., Die Abtreibung der Leibesfrucht. Berk Ärzte-Corr. 21. 
1. X. S. 312. Rucker, Beckenausgang-Meßinstrument. J. Am. m. Ass. 15. X. S. 1255. Runge, E., Geburtshilfe der Unfallstation. Med. Klin. 13. XL S. 1393.

20. XL S. 1422. Nr. 47, 48, 49 u. 50. Sachs, E., Zur Entwicklung des nachfolgenden Kopfes bei totem Kind.

Zbl. f. Gyn. 29. X. S. 1578. Schiffmann, Die Prophylaktische Indikation zur Sectio caesarea sowie

Bemerkungen über die Indikation zur Sectio caesarea überhaupt.

Zbl. f. Gyn. 22. X. S. 1527. Schönfeld, Experiment elle Untersuchungen über die Toxizität von

Plazenta-

lipoiden, mit Bezug auf die Ätiogenese der Plazentareklampsie.

Arch. f. Gyn. 115. H. 1. S. 80. Schwab, Xachtrag zu: Die Ur, sachen des uiistillbaren Erbrechens in der Schwangerschaft (in Xr. 27). Zbl. f. Gyn. 22. X. S. 1535.

228 Literatur-Verzeichois.

Scaglíone, Meningitis cerebrospinalis epidemica in graviditate. Riv.

d'pstet. gin. prat. III. Nr. 7. S. 275. Selllieim, Straffreiheit der Abtreibung. Dtsch. med. Woch. 3.

XI. S. 1334,

1366. Siefart, Kritische Bemerkungen über Abortbehandlung. Berl. klin. Woch.

14. XI. S. 1362. Silberstein, P., Raynaudsche Krankheit und Schwangerschaft. Z. f. G.

u. Geb. LXXXIV. H. 1. S. 208. Sippel, Über die Hüftgelenksluxation bei Neugeborenen und andern an-

geborenen Deformitäten. Munch, med. Woch. 23. IX. S. 1221. Slemons und Morriss,

Reststickstoff und Harnstoff im mütterlichen und

fötalen Blute zur Zeit der Geburt. B. Johns Hopkins H. XXVII.

Nr. 310. Samuel, Über Erleichterung der Geburt. Munch, med. Woch. 28. X. S. 1388. Spirito,

Nochmals über den Einfhiß des C'offein und des Caffeinfuses auf

die Uteruskontraktion. La Medicina pratiea. VI. fasc. 10. Spirito, Die Wirkung der Chininsalze

auf den überlebenden menschlichen

Uterus. Rasegna internat. di Clinica e Therap. II. Nr. 11. Stenzler, Zur Kasuistik des

Schiefhalses. Zbl. f. Gyn. 12. XI. S. 1635. Stoeckel, Über Placenta praevia. Z. f. ärztl.

Fortbildung. 21. Nr. 4. Stoeckel, Zu clem Artikel von A. Mühlberger: „Kritische Bemerkungen

zur

zeitweiligen Sterilisation der Fran" in Nr. 33 d. Zbl. f. G. Zbl. f. Gyn.

5. XI. S. 1617. Strakosch u. Anders, Beitrag zur Lehre vvn den Akardiern: über einen

Holoakardius Eumorphus. Arch. f. Gyn. 115. H. 2. S. 408. Telfair, Tötlicher Fall von

Uterusruptur. Am. J. obst. gyn. II. Nr. 4.

S. 443 u. 444. , Völlige Erblindung während der Schwangerschaft. Vorgetragen und

besprochen in den New York Academy of Med. 26. IV. Siehe Am. J.

obst. gyn. II. Nr. 4. Temesváry, N., Über ein sehr junges menschliches Ei in situ. Arch. f.

Gyn. 115. H. 1. S. .184. Thélien, Die Behandlung der Plac. praevia. Rev. méd. Suisse roman.

1921.

Februar. Trope, Encephalitis lethargica bei Schwangerschaft, Geburt und im

Wochenbett. Casopulèk. ceskych. 1921. Nr. 22. Tukermann, Placenta praevia und Landarzt. Th.

d. Gegenw. 1920. H. 12. Tuma, Therapie des fieberhaften Abortus. Casogin lèk. ceskych. Nr.

14.21. Umfrage über Behandlung des septischen Abortes. Winter, Franz, Ker-

mauner, Opitz, Stoerkel, Küstner. Med. Klin. 27. XL S. 1450. -

Hoehne, A. Mayer, v. Jaschke, Füth, Rissmann, Kupferberg, Baumm. 
Med. Klin. 4. XII. S. 1483. - Pehens, v. Franqué, Zangemeister.

11. XII. S. 1515. Vauverts, Abwarten oder Eingreifen bei komplizierten Abort en. Rev.

franc, de gynec. et d'obst. Sept. 20. Vercesi, Plazentaveränderungen bei Genitalentzündungen.

Fol. gynec.

XIII. fan. 2. 1920. Yinson, Ösophagusstriktur nach Schwangerschaftserbrechen. Surg. gyn.

obst. Oct. 21. S. 412.

Literatur-Verzeichnis. 229

Vilalaza, Herzinsuffizieiiz und "vaginaler Kaiserschnitt. Rev. espan. de

obst. y Ginèc. Nr. 63. 1921. Vitanza, Ophthalmia neonati. Riv. d'ost. gin. pratic. III. Nr. 7.

S. 288. Wachter, Beitrag zur Kasuistik und Therapie der Placenta praevia. Schweiz.

med. Woch. 1920. S. 618. Waegeli, Hysterektomie bei akuter Puerperalinfektion. Gyn. obst. IV.

H. 3. S. 243. Walter, Einklemmung des myomatösen Uterus in der Schwangerschaft.

Frauenarzt. 1920. S. 11. Warnekros, Zur Ligatur der großen Beckenvenen bei puerperaler

Pyämie.

(Ein Beitrag zur Prognose der puerperalen Infektionen. Munch, med.

Woch. 2. XII. S. 1514. Wedderhake, Der Amnesieschlaf. Munch, med. Woch. 23. IX. S. 1224.

Weinzierl, Erfahrungen rnit der Kaseontherapie. Dtsch. med. Woch.

22. IX. S. 1120. Weinzierl, Seltene Atiologie eines Kephalhämatom. Zbl. f. Gyn. 8. X.

S. 1441. Werner, Paul und Rud. Sieglbauer, Beitrag zur Frage: Herzfehler und

Schwangerschaft. Arch. f. Gyn. 115. H. 1. S. 41. Werner, P., Weiterer Beitrag zur Kenntnis der

Wärmefunktion bei gesundeii

und kranken Schwangeren und Entbundenen. Arch. f. Gyn. 115.

H. 1. S. 63. Wiegels, Über die Indikationen zum küiistlichem Abort bei Herz- und

Nierenkrankheiten. Berl. klin. Woch. 3. X. S. 1188. Williams, Whitridge. Histologische Studien an 50 nach Kaiserschnitt

entfemten Uteri. Johns Hopk. Hops. Bull. XXVIII. Nr. 321. Williams, Wh., Kritische Würdigung $21 \mathrm{j}^{1} / \mathrm{shriger} \mathrm{Erf} \mathrm{aiming} \mathrm{mit} \mathrm{Sectio}$

caesarea. Bull. Johns Hopk. H. XXXII. Nr. 364. Zaekerl, Ein Beitrag zur Klinik und Therapie

der Eklampsie. Arch. f.

Gyn. 115. H. 2. S. 264.

Gynäkologíe.

$\Lambda$ schner, Über einen eigenartigen Ovarialtumor aus der Gruppe der Folli-kulome. Nebst

auffallenden Menstruationsstörungen und einem bisher noch nicht beschriebenen anatomischen

Befuncl in Form einer gänseeigroßen, massiven Corpus-luteum-ähnlichen Bildung. Arch. f. Gyn.

115. H. 2. S. 350.

Bégouin, Die ,Aushöhlung” als Verfahren der abdominalen Totalexstir-pation bei im kleinen

Becken eingekeilten Tumoren. Gyn. obst. 1920. I. Nr. 5.

Bier, Reiz und Reizbarkeit. Ihre Bedeutung für die praktische Medizin. Munch, med. $\Lambda$ V'och.

25. XL S. 1521.

Brody, Uterussarkom, vom Endometrium ausgehend. Johns Hop. H. XXIX Nr. 329.

Cramer und Schiff, Hungerosteomalakie. Rv. med. Suisse rom. 20. Nr. 11.

Dalché, Myome und Retroflexio. Rev. franc, gyn. obst. Dec. 1920.

Döderlein, Th. J., 'Typen der Beckeninfektionen. Surg. gyn. obst. XXX. Nr. 6.

230

Literatur-Verzeichnis.

Dolansky, Das Coporus luteum als Diagnostikum und Therapeutiktim. 
Cas. lékar. 1921. Nr. 6. Eccles, Die Frühdiagnose akuter Abdominalerkrankung. Brit. m. J. Nr. 3149. Földes, Über die Terpentinbehandlung der Adnextumoren. Maggm. Orv. 21.

Nr. 9. Fiith, Beitrag zur Scheidenverletzung mit Chlorzink. Arch. f. Gyn. 115.

H, 2. S. 383. Graf, Die Ausrottung des Hamröhrenkrebses unter zeitweiligem Aufklappen der Schoßfuge. Zbl. f. Gyn. 10. XII. S. 1777. Häggström, Zahlenmäßige Analyse der Ovarien eines gesunden 22jährigen

Weibes. (Mengenbestimmung der verschiedenen Gebiete des Ovarialpa,renchym, der Follikel, der zweikernigen Eier, der Corpora atretica und Corpora lutea). Upsala Lake for Forhandl. XXVI. S. 5 u. 6.

1921. Hallopeau-Calleville, Linksseitige Inguinalhernie mit Sitz und Ovar. Bui.

mem. soc. anat. Paris 1920. Mai. Hisgen, Beitrag zur operativen Therapie der Harninkonkruenz. Zbl. f.

Gyn. 10. XII. S. 1783. Hellier, Osteomalakie mit Oophorektomie behandelt. Br. m. J. 1920. Okt. Hobbs, Akute Infektionen des Endometrium. Br. m. J. Nr. 3158. Hoffmann, Klaus, Über Sakralanästhesie. Z. f. ärztl. Fortb. 21. N. 22. Imbert, Neue Operation des kompletten Dammrisses. Am. J. surgery.

1920. Sept. Johnston und Brown, Fall von doppelter kongenitaler Hydronephrose.

Edinb. m. J. 1921. Juni. Kelling, Zur Beseitigimg der durch die Palpation des Abdomens ansgelösten

Bauchdeckenkontraktion. Munch, med. Woch. 9. XII. S. 1590. Klika, Die Gefahren der Pyelographie und ihre Verhütung. Cas. lékas.

cesk. 1920. Nr. 39 u. 40. Kossmann, ZurÄtiologie der Analfissuren bei Frauen. Zbl. f. Gyn. 10. IX.

S. 1286. Kritzler, Unzuverläßliche Fieberthermometer. Dtsch. med. Woch. 8. XII.

S. 1497. Kulenkampff, Zur allgemeinen Bauchdiagnostik. III. Was fühlen wir im

Bauch? Dtsch. med. Woch. 8. IX. S. 1056. Kaehner, Adenomyoma uteri exurrens. Am. J. med. sciences. Sept. 21.

S. 424. Kiilz, Zur Frage des Ersatzes von Blutverlusten durch Gummí-Kochsalzlösungen. Dtsch. med. Woch. 8. XII. S. 1493. Laíley u. Cruishanth, Komplementbindung bei der Gonorrhöediagnosy

bei Frauen. Surg. gyn. Obst. Oct. 21. S. 414. Lathrop, Anästhesie mit Äther und Ö1 per rectum. J. Am. m. Ass. 10. VII.

20. Lorin, Verkalkungen der Riesenmyome. Rev. franc, gyn. obst. Febr. 21. Lôwen, Über Erschlaffung des Sphinkter ani beim Douglasabszeß. Munch.

med. Woch. 18. XL S. 1484. Léry und Guillaume, Tube als Bruchinhalt. Bui. mem. soc. anat. Paris 1920.

Nr. 4.

Litcratur-Verzeiclmís.

231

Linzenmeier, Über Zystoskopie in der luftgefüllten Blase. Zbl. f. Gyn.

10. XII. S. 1786. Lory, Zwei Fälle von Hämatosalpinx mit Stíeldrehung. B. et mem. soe. anatom. Paris 1920. Mai. Ale Arthur, 1st der Chirurg jemals berechtigt gegen die anerkannte chirur-

gische Technik zu verstoßen bei der Behandlung maligner Neoplasmeu ?

Surg. Gyn. Obst. Oct. 21. S. 406. Magid, Ectropion abdominaler Eingeweide. Am. .1. obst. gyn. II. Xr. 4. 
S. 446. Vorgetragen und besprochen in der New York obst. Soc·

12. IV. 1921.

Marchand, Der gegenwärtige Stand der Entzündungsfrage. Dtsch. med.

Woch. 6. X. S. 1197. Martens, Über Ersatz der fehlenden Vagina durch Darin. Dtseh. med.

Woch.

13. X. S. 1226.

Alathes, Prolaps- und Retroflexionsfragen. Zbl. f. Gyn. 8. X. S. 1429. Mayer, A., Über abnorme Kontraktionsphänomene am Darm (segmentärer

Darmkrampf). Zbl. f. Gyn. 12. XI. 8. 1621. Mayiard, Bauchschmerz Mechanisrnus und klinische Bewertung. Brit.

m. J. Nr. 3151. Mans/eld, Über die Erfolge und Aussichten der Ovarientransplantat·ion.

Orvoskepzes 1921. Festschrift für Tauffer. Merhaut, Technik der kosmetischen Hautnaht.

Casopis lék. cesk. 1920.

Nr. 37. Meyer, Rob., Plattenepithelknötchen in hyperplastischen Driisen der

Korpusschleimhaut des Uterus und bei Karzinom. Arch. f. Gyn. 115.

H. 2. S. 394. - . Zur Kenntnís des Papilloma portionis uteri, insbesondere des Papilloma

verrucosum. Arch. f. Gyn. 115. H. 1. S. 167. - , Zur Bildung des Urnierenleistenbandes und zui • AdOnomyomlehre.

Arch. f. Gyn. 115. H. 1. S. 199. Michael, Zur künstliehen Scheidenbildung. Zbl. f. Gyn. 19. XI.

S. 1665. AJíkulicz-Radeckí, F. v., Die Bedeutung der Bezeichung Follikelatresie.

Zbl. f. Gyn. 12. XL S. 1636. Moinburg, Die intravenöse Atheinarkose. Dtsch. med. Woch. 13. X. S. 1228. Moerehen, Das Problem der Hysteric. Munch, med. Woch. 23. IX.

S. 1220. Mutter, Hugo, Verbreitung der Ceschlechtskrankheiten in Rheinhessen und Bordellfrage in Mainz. Munch, med1. Woch. 5. VIII. S. 973. Murray, Beziehungen

zwischen clem einzuführenden Ovarium und der

Geschlechtsbilclung. Johns Hop. H. Bui. Nr. 334. Nixon, Vereitertes Myoma uteri. J. Am. m. Ass. 1920. Nr. 251. Xorris u. Möekeberg, Diagnose der weiblichen Gonorrhöe durch Fiebermethoden. J. Am. m. Ass. Nj· 3. 1921. Norrín, Gonorrhöebehandlung bei L·okalisation im unteren Teil des Genito-

urinaltrakt. Surg. Gyn. obst. Sept. S. 308. Okabayashi, Radikale abdominale Hysterektomie wegen Uterushalskrebs,

Modifikation der Takayama-Operation. Surg. Gynec. Obst. Oct. 21.

S. 335.

Monatsschrift f. Gebiu-tshiilf'e u. (íynäkologie. Bel. LVח. Heft a 4.

232 Literatur-Verzeichnis.

O'Conor, Uber das Auswasclien der Xierenbecken: experiment ell e Studio.

J. Am. in. Ass. 1. X. S. 1088. Oelze, Untersuchungsmethoden uiid Diagnose der Erreger dor Geschlechts-

krankheiten. München, Lehmann 1920. Oxley u. J)ttndar, Fall von gonorrhöischer Mastitis. Br. m. .). Nov. 20. Palmen, 7)., Rudolf Steiners Anthroposophie und ihre Beziehnngen zur

Medizin. Dtsch. med. Woch. 18. VIII. S. 966. Parma, Zur Therapie der Hydroa aestivales. Oiská dermat. II. S. 44. 11)20. Patterson und Johns. Kurze Ûbersicht der modernen Methoden zur Diagnose

der Nierenstörungen. Ther. gaz. 1921. «Juni. Pencil, Über erne vor die äußere Hamröhrenmündung vorgefallene Ureter- 
zyste. Wien. klin. Woch. 10. XL S. 544. Peterson, Reuben, Pneumoperitoneum und

Rontgenology als Hilfsmittel

zu exakter Diagnose in Geburtshilfe und Gynäkologie. Am. J. ohst.

gyn. II. Xr. 4. S. 349. Pototzky, Das Pubertätsbasedowoid. Beit-rag zur Klinik der Hyper-

thyreosen im Pubert $1 / 8$ tsalter. Dtsch. med. Woch. X'r. 4. 21. Prinz, Orale Reiztherapie. Munch, med. Woch. 23. IX. S. 1215. Pujiulo, Das Urogenitalsystem. Rev. espan. de obst. y gin. 1921.

Xr. 04. Pusl, Die Behandlung der Zervixerkrankungen mit Hilíe von Zelluloid-

kapseln. Munch, med. Woch. 21. X. S. 1362. Rabboni, Die Wrightsche Vakzintherapie bei den

gonorrhöischen Krank-

heiten. Pass. d. obst. e. ginec. 20. Xi .1 3. Raefler, Die Hypnose in der Gynäkologie. Zbl. f. Gyn.

10. IX. S. 1274. Ransohoff-Dreyfoos, Gefahrvolle intraperitoneale Blutung aus einem

Uterusfibroid. Surg. gyn. obst. Sept. S. 296. Recasens, Kin Fall von Riesenmyom. Revista espan. obst. ginec. 1921.

Xr. 62. Reder, Kraurosis vulvae und Adenitis inguinalis maligna. Surg. gynee.

obst. Nov. 21. Xr. õ. S. 554. Reynolds, Gewisse Diätfaktoren für die Sterilität der Ratten. Am. .1. obst.

Gyn. II. Xr. 4. S. 379. Robinson, Yerletzungen des weiblichen Ureters. Brit. m. .1. Xr. 3149.

Rotter, II., Histogenese der malignen Geschwülste. Zbl. f. Krebs †'orsch.

P,d. IX. H. 3. Roy Me Clure, Bluttransfusion, Geschichte, Methoden, Gefahren, gegenwärtiger Stand. Bull. Johns Hopkins Hosp. XXVIII. Xr. 312. Rubin. Die nicht operative Peststellung dor Durehgängigkeit dcv Tuben

durch intrauterine Einblasung von Sauerstoff und Erzeugung eines

künstlichen Pneumoperitoneum. J. Am. m. Ass. L·XXV. Xr. 10. 20. Sandek, Das Okklusivpessar als Schiitzmittel gegeii die Gonorrhöe. Ceská

dermat. II. S. 41. Sanderson, Menstruation im Sclmlleben. Brit. med. •I. Xr. 31 IS. Schacht, Die Geschlechtsreife. Frauenarzt 1920. H. 9. Sehröder, R. u. Frieda Neuendorff-Viek, Der

menstruelle Zyklus hoi akut-

und chronisch-entziindlicher Adnexerkrankung (zugleich ein Bill!

vom Yerlauf der akuten und chronischen Endometritis interstitialis).

Arch. f. Gyn. 115. H. 1. S. 15. Schroder. R. u. E. A. Kuhlmann, Die Ulce > - ation der Vagina.

Zugleich

Literatur-A'erzeichnis.

23:î

Mitt $\beta$ ilung über je einen Fall von sog. Uleus rotundum und Ulcus

varicosum vaginae. Arch. f. Gyn. 115. H. 1. S. 145. Schroder, Ji., Zur Pat hogenese vm $<1$

Klinik des vaginal en Fluor. Zbl. f. G.

24. IX. S. 1350 und 1. X. 8. 1398. Schumann, Beobachtungen über Blutungen ovariellen und tuberk. II-

sprungs ohne ektopische Schwangerschaft. J. Am. m. Ass. 27. VIII.

»S. 692. Seil:, L., Primärie der Eizelle, Corpus hiteum, Menstruationszyklus und

Genese der Myome. Arch. f. Gyn. 115. H. 1. S. 1. Severance, Ein Ausflußanhänger fur das

Hvstoskop. .). Am. m. Ass.

27. VIII. S. 706. Siedentopf, Heilung einer doppelseitigen Hämatosalpinx (lurch Röntgenstrahlen. Zbl. f. Gyn. 10. IX. S. 1288. Stanca, Atresia vaginae puerp. dilatatio urethrae e Coita. Zbl. f. Gyn.

10. XII. S. 1788. Stein, Arth., Primäres Vulvakarzinom. Am. J. obst. and diseas of Worn. 
Nr. 4. 1919. Stein, A. u. Stewart, W-, Pneumoperitoneale Roiitgenstrahlendiagnose.

New York. Southworth Comp. 1921. Stein-, A., Syphilor@a vulvae. Surg. gyn. obst. XXXI. Bú. 'i. Swart:, Shohl u. Davis, Kultui'elle Eigentümlichkeiten (let· Gonokokken.

Bull. Johns Hopk. Hosp. XXXI. S. 358. Stephan, Bemerkungen zur Aetiologie und Therapie der Triehomonaskolpit·is.

Zbl. f. Gyn. 29. X. S. 1565. Stevens u. Heppner, Weibliche Gonorrhöe des unteren Urogenitalabschnittes.

J. Am. in. Ass. Nr. 22. 20. Trancu-Rainer, Außergewöhnlich große Luteinzyste. Zbl. f. Gyn. 26. XI.

S. 1702. Trebing, Über Ergopan. Zbl. f. Gyn. 15. X. S. 1494. Tnrán, Klimakterium und gichtische Konstitution. Gyoppées. 1920.

Xr. 16. Vineberg, Supracervikale vaginale Hysterektomie mit Interposition des

Zervixstumpfes bei Cystocele und. Verfall des vergrößerten Uterus.

Am. .1. obst. gyn. IT. Nr. 4. S. 368. Yitanza, Zur Prophylaxe des Uteruskarzinom. Rev. d'ost. ginec. pratica II I.

Nr. 9. S. 395. Yogi. Die Bekämpfung der postoperativen Urínverhaltung durch intravenöse Urotropininjektionen. Zbl. f. Gyn. 10. XII. S. 1781. Wallerstein, Zwei Fälle von künstlicher Scheidenbildung. Zbl. f. Gyn.

15. X. S. 1492. Watkins, Die Behandlung infizierter Bauchwunden durch das geschlossene Verfahren. J. Am. m. Ass. 27. VIII. S. 676. Wiegels, Über einen besoneren Fall von tot alem Dammriß. Zbl. f. Gyn.

17. XII. S. 1797. Willis, Klinische und experimentelle Beobachtungen über Salzwasserirrigation bei der Behandlung der diffusen Peritonitis. Surg. gyn.

obst. Oct. 21. S. 355. Winter, Die zunehmende Inoperabilität $d(>$ s Uteruskrebses und ib/re

Bekämpfung. Zbl. f. Gyn. 2. XII. S. 1723.

234

Litcratur-Verzeichnis.

Zietschmann, Über Funktionen des weiblichen Genitals bei Säugetier und Mensch.

Yergleichendes über die zystischen Prozesse der Brunst und Menstruation. Arch. f. Gyn. 115. H. 2. S. 201.

Zill, Zu $\gamma$ Frage der Heilbarkeit der weiblichen Gonorrhöe. Munch, med. Woch. 16. IX. S. 1183. Zweifel, P., Über die Bedeutung der Friihdiagnose fur die Dauerheilung des Gebärmutterkrebses. Munch, med. Woch. 23. IX. S. 1207.

Strahle $\pi$ therapie.

(iál, Die Strahlenbehandlung der Fibromyome und Metropathien. Orvos-

kepzes. 1921. Festschrift für Tauffer. -, Strahlenbehandlung der weiblichen Geschlechtsorgane.

Bndap. Kir.

Oi voßgyer. 1920. 27. XL Knox, Behandlung mit Strahlen und Radium. Würdigung dieses

Mittels.

Edinburgh m. J. $\Lambda$ Гai Juni 1921. Lome, Zur Kritik dev Ozonbestimmung. (Ein Beit rag zm•

Frage der Gas-

vergiftung irn Röntgeiizimmer.) Munch, med. Woch. 25. XI. S. 1519. Markovits, Die

Röntgentherapie in der Gynäkalogie. Gyógyaszes. 1921.

Xr. 12. Pircle, t $\tau$ ber Radiumtherapie. Br. rn. .). Xr. 3135. Scott, Kombinierte Strahlenbehandlung bei bösartigen Erkrankungen.

Br. in. .J. Xr. 3152. Seilz, A., und Vey, Die Diatherminbehandlung der weiblichen Brust. 
Zbl. f. Gyn. 3. XII. S. 1744. Steltner, Fine einfache Methode •luv planmäßigen Röntgentiefendosimetrie.

Munch, med. Woch. 2. XII. S. 1559. Zander, Rudimentare Radiumdosiermg. Arch. f. Gyn. 115. H. 2. S. 253. 